\title{
A short course about fitting models with the scipy .optimize module
}

\section{Ariel Rokem $^{1}$}

DOI: $10.21105 /$ jose. 00016

\section{Software}

- Review ¿

- Repository ca

- Archive cta

Submitted: 26 April 2018

Published: 04 July 2018

\section{License}

Authors of papers retain copyright and release the work under a Creative Commons Attribution 4.0 International License (CC-BY).
1 The University of Washington eScience Institute

\section{Summary}

Fitting models and testing the match of the models to the measured data is a fundamental activity in many fields of science. This short (approximately 3-hour) course (available at: https://github.com/arokem/scipy-optimize) aims to teach participants to use the Scipy library's optimize module to fit models to data (Jones et al. 2001). Using data from a psychology experiment (Rokem and Landau 2016) as an example, the course motivates the use of explicit mathematical models to explain and predict data and compares linear models and non-linear models. The core of the lesson focuses on fitting a curve with the curve_fit function. The course also introduces the idea of model comparison with cross-validation for evaluation and selection between non-nested non-linear models.

\section{Statement of need}

Model fitting is useful in many different fields of research, but optimization for model fitting is not a topic that is usually covered in introductory statistics or computing classes in many fields (e.g., psychology). This course fills an existing need for hands-on curriculum that goes beyond the topics taught in introductory computing workshops, such as Software Carpentry, providing material for follow-up workshops on advanced/intermediate topics. The target audience for this course is researchers or students with some programming knowledge (e.g., having participated in a Software Carpentry workshop beforehand).

\section{Learning objectives}

In addition to the general objectives of this lessson, specific learning objectives are defined for each part of the lesson:

Part 1:

- Learners can define what a model is.

- Learners can define model parameters and model fitting.

- Learners can restate the benefits of modeling

- Learners can explain the utility of modeling applied to their data.

Part 2:

- Learners can identify a linear model.

- Learners can use numpy to fit a linear model to data

- Learners can evaluate a model using model residuals.

Part 3: 
- Learners can identify a nonlinear model, and discuss the differences between linear and nonlinear models

- Learners can use scipy.optimize to fit a nonlinear model to data.

- Learners can calculate and display model residuals for nonlinear models

Part 4:

- Learners can define and identify overfitting.

- Learners can implement split-half cross-validation to evaluate model error.

\section{Description of the module}

This course originated from a blog post that extended the final exercise for the Software Carpentry instructor training (2013). It was then also taught as a remote online workshop, as part of a series of remote workshops organized by the UC Davis Data Intensive Biology Lab.

The data used in the course comes from an experiment in visual neuroscience (Rokem and Landau 2016), but follows a format that is similar to data formats in many fields of science: repeated observations of a binary response (dependent) variable, for which the probability of a particular response depends on a known (independent) input variable. At the outset of the course, learners follow along as we read the data from a comma-separated file, plot the data, and transform it into a plot of probability of response as a function of input. The core of the course is a series of lessons on modeling these data: initially using a linear model, and then using non-linear models. This allows learners to understand and describe the distinction between these. The course introduces a functional form for these data and then uses the curve_fit function from the scipy.optimize module to fit these functional forms, with a sum-of-squared-errors objective function. The course also briefly introduces model comparison using cross-validation and motivates this with an example of over-fitting.

\section{Usage}

To use these instructional materials, it is recommended that the instructor type out the code in an interactive environment, such as a Jupyter notebook, while learners follow along on their own machines. For this purpose, prerequisites and setup instructions are provided on the first page of the lesson.

\section{Example of instruction}

A video of an example of instruction using this lesson is available on YouTube

\section{References}

Jones, Eric, Travis Oliphant, Pearu Peterson, and others. 2001. "SciPy: Open Source Scientific Tools for Python." http://www.scipy.org/.

Rokem, Ariel, and Ayelet Nina Landau. 2016. "The Interaction of Orientation-Specific Surround Suppression and Visual-Spatial Attention." bioRxiv. https://doi.org/10.1101/ 091553. 